ANNALES

POLONICI MATHEMATICI

$96.1(2009)$

\title{
Generalized iterated function systems, multifunctions and Cantor sets
}

\author{
by Maciej Klimek (Uppsala) and Marta Kosek (Kraków)
}

\begin{abstract}
Using a construction similar to an iterated function system, but with functions changing at each step of iteration, we provide a natural example of a continuous one-parameter family of holomorphic functions of infinitely many variables. This family is parametrized by the compact space of positive integer sequences of prescribed growth and hence it can also be viewed as a parametric description of a trivial analytic multifunction.
\end{abstract}

1. Introduction. For any given function $\rho: \mathbb{N} \rightarrow \mathbb{N}$, we say that the growth of a sequence of natural numbers $\sigma: \mathbb{N} \rightarrow \mathbb{N}$ is prescribed by $\rho$ if $\sigma \leq \rho$. The set $\Sigma_{\rho}$ of all such sequences can be easily turned into a compact space if two sequences are regarded as close provided that sufficiently many of their initial terms coincide. Let $\mathcal{A}(E)$ denote the Banach space of continuous affine operators on a complex Banach space $E$. Consider a matrix $T=\left[T_{n, j}\right]_{n \geq 1,1 \leq j \leq \rho(n)}$ whose entries belong to the open unit ball in $\mathcal{A}(E)$. The matrix has infinitely many finite length rows, possibly of different sizes dictated by the function $\rho$. By a column of $T$ we understand any sequence of the form $T_{\operatorname{col}(\sigma)}=\left\{T_{n, \sigma(n)}\right\}_{n \geq 1}$, where $\sigma \in \Sigma_{\rho}$. Since the entries of $T$ are contractions, if we assume that their contraction ratios are uniformly bounded by a constant smaller than 1 , then independent of the choice of $z \in E$, the sequence $\left(T_{1, \sigma(1)} \circ \cdots \circ T_{n, \sigma(n)}\right)(z)$ converges to a limit, say $f\left(T_{\operatorname{col}(\sigma)}\right)$. We will denote by $\Omega_{\rho}$ the set of all $T$ of this type. One of our objectives is to show that the mapping

$$
\Sigma_{\rho} \times \Omega_{\rho} \ni(\sigma, T) \mapsto f\left(T_{\operatorname{col}(\sigma)}\right) \in E
$$

is continuous and holomorphic with respect to $T$. Furthermore, the set-valued mapping

$$
\Omega_{\rho} \ni T \mapsto\left\{f\left(T_{\operatorname{col}(\sigma)}\right): \sigma \in \Sigma_{\rho}\right\} \in \operatorname{Comp}(E)
$$

2000 Mathematics Subject Classification: Primary 32H02, 32A12, 32H50; Secondary 46G20, 54C50, 26E25.

Key words and phrases: holomorphic mappings, analytic multifunctions, set-valued functions, affine contractions, iterations, generalized Cantor sets. 
is upper semicontinuous and has values in the set $\operatorname{Comp}(E)$ of all compact subsets of $E$. In particular, if $E=\mathbb{C}^{N}$, we get an example of a so-called trivial analytic multifunction.

The modern concept of an analytic multifunction whose values are compact subsets of $\mathbb{C}$ was conceived by Oka over 70 years ago in [14]. The idea was picked up later by Nishino [13] and Yamaguchi ([22], [23]), but otherwise - despite the widespread recognition of other discoveries made by Oka - this particular notion went largely unnoticed for many decades. The interest in analytic multifunctions was reignited in the early 1980's, largely due to Słodkowski introducing different equivalent descriptions of such functions and leading the way to multi-dimensional generalizations (see in particular [17] and [18]). A general systematic approach was developed nearly at the same time by Ransford (see e.g. [16]). Initially much of the interest was linked to spectral theory in Banach algebras (see e.g. [8], [19], [20]) but later it was complex dynamics that yielded new classes of analytic multifunctions (see [2], [9], [10], [11], [1], [12], [3]).

Trivial analytic multifunctions - defined as unions of graphs of holomorphic functions - are important not just as basic examples. Their significance is shown in Słodkowski's theorem stating that any (strongly) analytic multifunction can be approximated by a decreasing sequence of locally trivial functions [21]. At the same time, specific one-parameter families of holomorphic functions have become an object of interest as a result of research on holomorphic motions in one complex variable (see e.g. [3]).

The research described in this paper has been motivated partly by the background outlined above and - in particular - by the results presented in [3] concerning iterated function systems in $\mathbb{C}$ viewed in the context of analytic multifunctions. Also, the intention was to investigate how polynomial based constructions used in [11] and [12] translate into the case of affine operators.

The organization of the paper is as follows. In Section 2 we introduce notation and present the basic iteration scheme for sequences of affine operators in a Banach space. Sections 3, 4 and 5 are devoted to various properties of attractors of iterated function systems based on families of affine mappings. In Section 6 we present the example announced above. The final section contains remarks about Cantor sets relevant in this context. It is worth mentioning that every generalized Cantor set can occur as the attractor in our construction, which was not the case for classical IFSs.

2. Sequences of continuous affine operators. Let $(E,\|\|)$ be a complex Banach space. Denote by $\mathcal{L}(E)$ the space of bounded linear operators on $E$, furnished with the usual operator norm, and by $\mathcal{A}(E)$ the space of continuous affine operators on $E$. Each such operator $T: E \rightarrow E$ has the 
decomposition $T=\widehat{T}+T(0)$, where $\widehat{T} \in \mathcal{L}(E)$. Hence $\mathcal{A}(E)=\mathcal{L}(E) \oplus E$ and the natural norm in $\mathcal{A}(E)$ is given by the formula $\|T\|=\|\widehat{T}\|+\|T(0)\|$.

We will consider Banach spaces of sequences or different types of matrices of affine operators. If $\mathcal{S}$ is a nonempty set, then

$$
\ell^{\infty}(\mathcal{S}, \mathcal{A}(E))=\left\{T: \mathcal{S} \ni s \mapsto T_{s} \in \mathcal{A}(E): \sup _{s \in \mathcal{S}}\left\|T_{s}\right\|<\infty\right\}
$$

endowed with the norm $\|T\|=\sup _{s \in \mathcal{S}}\left\|T_{s}\right\|$ is a complex Banach space. For $T=\left(T_{s}\right)_{s \in \mathcal{S}} \in \ell^{\infty}(\mathcal{S}, \mathcal{A}(E))$, we define $\widehat{T}=\left(\widehat{T}_{s}\right)_{s \in \mathcal{S}}$. It will be useful to consider the open set

$$
\Omega_{\mathcal{S}}=\left\{T \in \ell^{\infty}(\mathcal{S}, \mathcal{A}(E)):\|\widehat{T}\|<1\right\} .
$$

In this section we are interested in sequences, so we take $\mathcal{S}=\mathbb{N}$ in (1) and (2). We will need an enhanced version of Banach's Contraction Principle [11, see Lemma 4.5 and its proof].

Lemma 2.1 (Enhanced version of Banach's Contraction Principle). Let $(X, \varrho)$ be a complete metric space and let $\left(H_{n}\right)_{n \geq 1}$ be a sequence of contractions of $X$ with contraction ratios not greater than $L<1$. If

$$
M=\sup _{n \geq 1} \varrho\left(H_{n}(x), x\right)<\infty
$$

for each $x \in X$, then there exists a unique point $c$ in $X$ such that the sequence $\left(H_{1} \circ \cdots \circ H_{n}\right)_{n \geq 1}$ converges pointwise to $c$.

Furthermore, if $K \subset X$ is such that

$$
M_{K}=\sup _{x \in K} \sup _{n \geq 1} \varrho\left(H_{n}(x), x\right)<\infty,
$$

then the convergence is uniform on the set $K$.

As a consequence we easily get the following statement.

Lemma 2.2. If $T \in \Omega_{\mathbb{N}}$, then the sequence $\left(T_{1} \circ \cdots \circ T_{n}\right)_{n \in \mathbb{N}}$ converges uniformly on bounded sets to a constant mapping.

Proof. Note first that $T_{n}, n \in \mathbb{N}$, is a contraction with contraction ratio not greater than $\|\widehat{T}\|<1$, since $\left\|T_{n}(z)-T_{n}(w)\right\|=\left\|\widehat{T}_{n}(z)-\widehat{T}_{n}(w)\right\| \leq$ $\|\widehat{T}\|\|z-w\|$ for any $z, w \in E$. Furthermore,

$$
\left\|T_{n}(z)-z\right\| \leq\left\|\widehat{T}_{n}(z)\right\|+\left\|T_{n}(0)\right\|+\|z\| \leq(\|\widehat{T}\|+1)\|z\|+\|T\|
$$

for any $z \in E$ and $n \in \mathbb{N}$, so the conclusion is a direct consequence of Lemma 2.1 .

The above lemma shows the existence of the limit of the sequence $\left(T_{1} \circ \cdots \circ T_{n}\right)_{n \in \mathbb{N}}$ but says nothing about its dependence on the original sequence $T$. It turns out that this dependence is holomorphic, as shown in the following theorem. 
THEOREM 2.3. If

$$
f_{n}(T, z)=\left(T_{1} \circ \cdots \circ T_{n}\right)(z), \quad(T, z) \in \Omega_{\mathbb{N}} \times E,
$$

then the sequence of holomorphic mappings $\left(f_{n}\right)_{n \geq 1}$ converges locally uniformly in $\Omega_{\mathbb{N}} \times E$ to a holomorphic mapping $f$, which is independent of $z$. The rate of convergence satisfies the estimate

$$
\left\|f(T)-f_{n}(T, z)\right\| \leq(2\|z\|+\|T\|) \frac{\|\widehat{T}\|^{n}}{1-\|\widehat{T}\|}, \quad(T, z) \in \Omega_{\mathbb{N}} \times E .
$$

Furthermore, if $T=\left(T_{n}\right)$ is such that all $T_{n}$ are bijective then

$$
\lim _{n \rightarrow \infty}\left\|\left(T_{1} \circ \cdots \circ T_{n}\right)^{-1}(w)\right\|=\infty
$$

for any $w \in E \backslash\{f(T)\}$.

Proof. By Lemma 2.2 we can define

$$
f(T)=\lim _{n \rightarrow \infty} f_{n}(T, z), \quad(T, z) \in \Omega_{\mathbb{N}} \times E .
$$

We have

$$
\begin{aligned}
\left\|f_{n+k}(T, z)-f_{n}(T, z)\right\| & \leq \sum_{j=1}^{k}\left\|f_{n+j}(T, z)-f_{n+j-1}(T, z)\right\| \\
& \leq \sum_{j=1}^{k}\|\widehat{T}\|^{n+j-1}\left\|T_{n+j}(z)-z\right\| \\
& \leq(2\|z\|+\|T\|) \sum_{j=1}^{k}\|\widehat{T}\|^{n+j-1} .
\end{aligned}
$$

By letting $k$ go to infinity we obtain the estimate (3).

Assume now that $(T, z) \in \Omega_{\mathbb{N}} \times E$ and $T=\left(T_{n}\right)$ is such that all $T_{n}$ are bijective. Note that the inverses are continuous. First we will show that if the set

$$
S_{z}=\left\{\left(T_{1} \circ \cdots \circ T_{n}\right)^{-1}(z): n \in \mathbb{N}\right\}
$$

is bounded, then $z=f(T)$. Suppose that $z \neq f(T)$. If $n$ is sufficiently large, then $\left\|f(T)-f_{n}(T, w)\right\|<\|f(T)-z\|$ for all $w \in S_{z}$. In particular, this should be true for $w=\left(T_{1} \circ \cdots \circ T_{n}\right)^{-1}(z)$, which is clearly impossible.

Suppose now that (4) does not hold. Then there exist a $w \in E \backslash\{f(T)\}$ and an increasing sequence $\left(n_{k}\right)$ of integers such that the set

$$
\left\{\left(T_{1} \circ \cdots \circ T_{n_{k}}\right)^{-1}(w): k \in \mathbb{N}\right\}
$$

is bounded. We can now apply the above argument to the mappings

$$
T_{1} \circ \cdots \circ T_{n_{1}}, T_{n_{1}+1} \circ \cdots \circ T_{n_{2}}, \ldots, T_{n_{k-1}+1} \circ \cdots \circ T_{n_{k}}, \ldots
$$


instead of $T_{1}, T_{2}, \ldots, T_{k}, \ldots$. Since the fixed point $f(T)$ is exactly the same for the new sequence of maps, we conclude that $w=f(T)$, which is not the case.

If $T \in \Omega_{\mathbb{N}}$, by Banach's Contraction Principle all the mappings $T_{1} \circ \cdots \circ T_{n}$ have unique fixed points. It turns out that $f(T)$ is the limit of those points.

COROLlary 2.4. If $T \in \Omega_{\mathbb{N}}$ and $a_{n}$ is the fixed point of $T_{1} \circ \cdots \circ T_{n}$, $n \in \mathbb{N}$, then

$$
f(T)=\lim _{n \rightarrow \infty} a_{n}
$$

Proof. We have

$$
\left\|a_{n}\right\|=\left\|\left(\widehat{T}_{1} \circ \cdots \circ \widehat{T}_{n}\right)\left(a_{n}\right)+\left(T_{1} \circ \cdots \circ T_{n}\right)(0)\right\| \leq\|\widehat{T}\|^{n}\left\|a_{n}\right\|+n\|T\|,
$$

hence by (3),

$$
\left\|f(T)-a_{n}\right\|=\left\|f(T)-f_{n}\left(T, a_{n}\right)\right\| \leq\left(2 \frac{n\|T\|}{1-\|\widehat{T}\|^{n}}+\|T\|\right) \frac{\|\widehat{T}\|^{n}}{1-\|\widehat{T}\|} .
$$

3. Bounded families of affine operators. Let $\operatorname{Bdd}(E)$ be the family of nonempty closed bounded subsets of $E$, and $\operatorname{Comp}(E)$ the subfamily of nonempty compact subsets of $E$. Denote by $d_{\mathrm{H}}$ the Hausdorff metric associated with the norm \|\| on $\operatorname{Bdd}(E)$. It is well known that $\left(\operatorname{Bdd}(E), d_{\mathrm{H}}\right)$ is a complete metric space and $\operatorname{Comp}(E)$ is a closed subset of $\operatorname{Bdd}(E)$.

Consider now a bounded family $\mathcal{F}$ in $\mathcal{A}(E)$ and let $M=\sup \{\|Q\|$ : $Q \in \mathcal{F}\}$. If $K \in \operatorname{Bdd}(E)$ and $R=\sup \{\|z\|: z \in K\}$, then $\sup \{\|Q(z)\|:$ $z \in K\} \leq(R+1) M$ for each $Q \in \mathcal{F}$. Thus the mapping

$$
\Phi_{\mathcal{F}}: \operatorname{Bdd}(E) \ni K \mapsto \Phi_{\mathcal{F}}(K)=\overline{\bigcup_{Q \in \mathcal{F}} Q(K)} \in \operatorname{Bdd}(E)
$$

is well defined.

It would be interesting to know whether $\Phi_{\mathcal{F}}$ (and more precisely its restriction) could be viewed as a mapping from $\operatorname{Comp}(E)$ to $\operatorname{Comp}(E)$.

Proposition 3.1. If the closure of the bounded family $\mathcal{F}$ in $\mathcal{A}(E)$ is compact, then

$$
\Phi_{\mathcal{F}}: \operatorname{Comp}(E) \ni K \mapsto \Phi_{\mathcal{F}}(K)=\bigcup_{Q \in \overline{\mathcal{F}}} Q(K) \in \operatorname{Comp}(E) .
$$

Proof. Pick $K \in \operatorname{Comp}(E)$, and put $R=\sup \{\|z\|: z \in K\}$ and $M=$ $\sup \{\|Q\|: Q \in \mathcal{F}\}$. We need to show that

$$
\bigcup_{Q \in \overline{\mathcal{F}}} Q(K)=\overline{\bigcup_{Q \in \mathcal{F}} Q(K)}
$$


$(\subset)$ Let $Q$ be the limit of a sequence $\left(Q_{\nu}\right) \subset \mathcal{F}$. Then for any $z \in K$, $\left\|Q_{\nu}(z)-Q(z)\right\| \leq\left\|\widehat{Q}_{\nu}-\widehat{Q}\right\|\|z\|+\left\|Q_{\nu}(0)-Q(0)\right\| \leq(R+1)\left\|Q_{\nu}-Q\right\| \rightarrow 0$.

(つ) Take $z \in \bar{\bigcup}\{Q(K): Q \in \mathcal{F}\}$. Then there exist sequences $\left(Q_{\nu}\right) \subset \mathcal{F}$ and $\left(w_{\nu}\right) \subset K$ such that $z_{\nu}=Q_{\nu}\left(w_{\nu}\right) \rightarrow z$. Since $K$ is compact, by taking a subsequence if needed, we may assume that $w_{\nu} \rightarrow a \in K$. As $\overline{\mathcal{F}}$ is also compact, we may assume again that $Q_{\nu} \rightarrow Q \in \overline{\mathcal{F}}$. Note that $\|\widehat{Q}\| \leq\|Q\| \leq M$.

Take $\varepsilon>0$. For $\nu$ large enough we have

$$
\begin{aligned}
\left\|z-z_{\nu}\right\| & <\varepsilon / 3, \\
\left\|z_{\nu}-Q\left(w_{\nu}\right)\right\| & \leq\left\|\widehat{Q}_{\nu}-\widehat{Q}\right\|\left\|w_{\nu}\right\|+\left\|Q_{\nu}(0)-Q(0)\right\| \leq(R+1)\left\|Q_{\nu}-Q\right\| \\
& <\varepsilon / 3, \\
\left\|Q\left(w_{\nu}\right)-Q(a)\right\| & =\left\|\widehat{Q}\left(w_{\nu}\right)-\widehat{Q}(a)\right\| \leq\|\widehat{Q}\|\left\|w_{\nu}-a\right\| \leq M\left\|w_{\nu}-a\right\| \\
& <\varepsilon / 3 .
\end{aligned}
$$

Therefore $\|z-Q(a)\|<\varepsilon$ by the triangle inequality and thus $z=Q(a) \in$ $Q(K)$, since $\varepsilon>0$ can be arbitrarily small.

It remains to verify that $\Phi_{\mathcal{F}}(K)$ is compact. Take a sequence $\left(z_{\nu}\right) \subset$ $\bigcup\{Q(K): Q \in \overline{\mathcal{F}}\}$. As before there exist sequences $\left(Q_{\nu}\right) \subset \overline{\mathcal{F}}$ and $\left(w_{\nu}\right) \subset K$ such that $z_{\nu}=Q_{\nu}\left(w_{\nu}\right)$. In view of the compactness of $K$ and $\overline{\mathcal{F}}$ we may assume that $w_{\nu} \rightarrow w \in K$ and $Q_{\nu} \rightarrow Q \in \overline{\mathcal{F}}$, therefore $z_{\nu} \rightarrow Q(w) \in \Phi_{\mathcal{F}}(K)$ as $\nu \rightarrow \infty$.

One can pose a natural question whether the assumption of Proposition 3.1 is really necessary for $\Phi_{\mathcal{F}}$ to map $\operatorname{Comp}(E)$ into $\operatorname{Comp}(E)$. The following easy example shows that it indeed is.

ExAmple 3.2. Take $E=\ell^{\infty}\left(=\ell^{\infty}(\mathbb{N}, \mathbb{C})\right)$ and let $\left(e^{n}\right)_{n \in \mathbb{N}}$ be its canonical basis $\left(e_{k}^{n}=\delta_{n k}\right.$, the Kronecker delta). Consider $\mathcal{F}=\left\{h_{n}: n \in \mathbb{N}\right\}$, where $h_{n}(z)=\frac{1}{2} z+e^{n}$. Then $\mathcal{F}$ is closed and bounded and

$$
\Phi_{\mathcal{F}}(\{0\})=\bigcup_{h \in \mathcal{F}} h(\{0\})=\bigcup_{h \in \overline{\mathcal{F}}} h(\{0\})=\left\{e^{n}: n \in \mathbb{N}\right\} \in \operatorname{Bdd}(E) \backslash \operatorname{Comp}(E) .
$$

We now turn our attention to bounded families of affine contractions.

Proposition 3.3. Assume that the family $\mathcal{F} \subset \mathcal{A}(E)$ is bounded and $m=\sup \{\|\widehat{Q}\|: Q \in \mathcal{F}\}<1$. Then the mapping $\Phi_{\mathcal{F}}$ defined by formula (5) is a contraction with ratio $m$. It has a unique fixed point. Moreover, if the closure of the bounded family $\mathcal{F}$ in $\mathcal{A}(E)$ is compact, the fixed point is compact too.

Proof. Pick $K, L \in \operatorname{Bdd}(E)$. If $z \in \Phi_{\mathcal{F}}(K)$ and $\varepsilon>0$, then $\|z-Q(\zeta)\|<\varepsilon$ for some $Q \in \mathcal{F}$ and $\zeta \in K$. Let $\xi \in L$ be such that $\|\zeta-\xi\|<\operatorname{dist}(\zeta, L)+\varepsilon$. 
Then

$$
\begin{aligned}
\operatorname{dist}\left(z, \Phi_{\mathcal{F}}(L)\right) & \leq\|z-Q(\xi)\| \leq \varepsilon+\|Q(\zeta)-Q(\xi)\| \leq \varepsilon+\|\widehat{Q}\|\|\zeta-\xi\| \\
& \leq(m+1) \varepsilon+m \operatorname{dist}(\zeta, L) \leq(m+1) \varepsilon+m d_{\mathrm{H}}(K, L) .
\end{aligned}
$$

Letting $\varepsilon \rightarrow 0$, we obtain $\operatorname{dist}\left(z, \Phi_{\mathcal{F}}(L)\right) \leq m d_{\mathrm{H}}(K, L)$. A similar estimate for $\operatorname{dist}\left(w, \Phi_{\mathcal{F}}(K)\right), w \in L$, yields

$$
d_{\mathrm{H}}\left(\Phi_{\mathcal{F}}(K), \Phi_{\mathcal{F}}(L)\right) \leq m d_{\mathrm{H}}(K, L) .
$$

By the Banach Contraction Principle, $\Phi_{\mathcal{F}}$ has a unique fixed point. By Proposition 3.1, under the assumption of the "moreover" part, the unique fixed point is compact as it is also the unique fixed point of the restriction of $\Phi_{\mathcal{F}}$ to $\operatorname{Comp}(E)$ given by formula (6).

We will need some special cases of Proposition 3.3. The following corollary is straightforward.

Corollary 3.4. Assume that the family $\mathcal{F} \subset \mathcal{A}(E)$ is bounded and $m=$ $\sup \{\|\widehat{Q}\|: Q \in \mathcal{F}\}<1$. If either the dimension of $E$ or the bounded family $\mathcal{F} \subset \mathcal{A}(E)$ is finite, then the mapping $\Phi_{\mathcal{F}}$ defined by $(5)$ is a contraction with ratio $m$ and has a unique compact fixed point.

Proof. If the family $\mathcal{F}$ is finite, then it is closed and compact.

Now assume that the dimension of the Banach space $E$ is finite. Then the space $\mathcal{A}(E)$ is also of finite dimension and thus the closure of the family $\mathcal{F}$ is compact.

4. Attractors of affine mapping matrices. In this section we will discuss two spaces given by formula (1). In a more general setting we take $\ell^{\infty}\left(\mathbb{N}^{2}, \mathcal{A}(E)\right)$. Another possibility is to take $\ell^{\infty}\left(\mathbb{N}_{\rho}, \mathcal{A}(E)\right)$, where for a fixed function $\rho: \mathbb{N} \rightarrow \mathbb{N}$ we define the index set

$$
\mathbb{N}_{\rho}=\left\{(n, j) \in \mathbb{N}^{2}: j \leq \rho(n)\right\} .
$$

Note that if the fixed function is constant, $\rho \equiv k$, then $\mathbb{N}_{\rho}=\mathbb{N} \times\{1, \ldots, k\}$.

Our objects $\ell^{\infty}\left(\mathbb{N}^{2}, \mathcal{A}(E)\right)$ and $\ell^{\infty}\left(\mathbb{N}_{\rho}, \mathcal{A}(E)\right.$ ) (and in the special case also $\left.\ell^{\infty}(\mathbb{N} \times\{1, \ldots, k\}, \mathcal{A}(E))\right)$ are spaces of matrices (with infinite or finite rows) of continuous affine operators. From now on we will use the letter $\Omega$ to denote either the open set $\Omega_{\mathbb{N}^{2}}$ or, respectively, $\Omega_{\mathbb{N}_{\rho}}$ (see $\left.(2)\right)$, since the construction is the same in both cases.

Let us now take $T=\left[T_{n, j}\right] \in \Omega$. Let $T_{n}$ denote the (unordered) set of all affine operators in the $n$th row of $T$. For any $n \geq 1$, the family $T_{n}$ is bounded by $\|T\|$ in $\mathcal{A}(E)$ and moreover $\sup \left\{\|\widehat{Q}\|: Q \in T_{n}\right\} \leq\|\widehat{T}\|<1$. Hence, by Proposition $3.3, \Phi_{T_{n}}$ is a contraction with ratio at most $\|\widehat{T}\|$. Note that if we want all families $T_{n}$ to be finite (we will need this for some results), we have to consider the space $\ell^{\infty}\left(\mathbb{N}_{\rho}, \mathcal{A}(E)\right)$. 
Proposition 4.1. If $T \in \Omega$, then the sequence $\left(\Phi_{T_{1}} \circ \cdots \circ \Phi_{T_{n}}\right)_{n \geq 1}$ converges pointwise to a constant mapping.

Proof. It suffices to verify the last assumption of the enhanced version of Banach's Contraction Principle (Lemma 2.1). Pick $K \in \operatorname{Bdd}(E)$ and put $R=\sup \{\|z\|: z \in K\}$. Then $\sup \left\{\|w\|: w \in \Phi_{T_{n}}(K)\right\} \leq(R+1)\|T\|$ and therefore

$$
\sup _{n \geq 1} d_{\mathrm{H}}\left(K, \Phi_{T_{n}}(K)\right) \leq R+(R+1)\|T\| .
$$

We denote by $A(T)$ the value of the constant mapping from Proposition 4.1. Note that if either the dimension of $E$ is finite or $T \in \Omega_{\mathbb{N}_{\rho}}$, then the set $A(T)$ is compact.

Moreover, if $T \in \Omega$ has the same finite rows (i.e. $T_{n}=\mathcal{F}$ for all $n \in \mathbb{N}$ and $\mathcal{F} \subset \mathcal{A}(E)$ is finite), then $A(T)$ is exactly the unique invariant compact set of $\mathcal{F}$ (an iterated function system of contractions) from Hutchinson's survey [7]. If $E=\mathbb{C}$, such invariant sets (not only for finite but also for countable families $\mathcal{F}$ ) can be considered as special cases of attractors studied in [3]. Therefore we will call $A(T)$ the attractor of the matrix $T$. Note that if all entries of $T$ have the same fixed point $a$ (for example if all entries are linear, their fixed points are equal to zero), then $A(T)=\{a\}$.

Let us now look at some properties of attractors.

Lemma 4.2. Let $T$ be in $\Omega$. If $K \in \operatorname{Bdd}(E)$ is such that $Q(K) \subset K$ for any $Q \in T_{n}$ and $n \in \mathbb{N}$, then

$$
A(T)=\bigcap_{n \geq 1}\left(\Phi_{T_{1}} \circ \cdots \circ \Phi_{T_{n}}\right)(K) .
$$

Proof. We have $\Phi_{T_{n}}(K) \subset K$ for all $n \in \mathbb{N}$, hence

$$
\begin{aligned}
& \Phi_{T_{1}}(K) \subset K, \\
& \Phi_{T_{1}}\left(\Phi_{T_{2}}(K)\right) \subset \Phi_{T_{1}}(K) \subset K, \\
&\left(\Phi_{T_{1}} \circ \cdots \circ \Phi_{T_{n-1}}\right)\left(\Phi_{T_{n}}(K)\right) \subset\left(\Phi_{T_{1}} \circ \cdots \circ \Phi_{T_{n-1}}\right)(K) \subset \cdots \subset K .
\end{aligned}
$$

The sequence $\left(\left(\Phi_{T_{1}} \circ \cdots \circ \Phi_{T_{n}}\right)(K)\right)_{n \in \mathbb{N}}$ is decreasing in $\operatorname{Bdd}(E)$ to the limit

$$
\bigcap_{n \geq 1}\left(\Phi_{T_{1}} \circ \cdots \circ \Phi_{T_{n}}\right)(K),
$$

which by Proposition 4.1 must be equal to $A(T)$.

For $R>0$, we denote by $\mathbb{B}_{R}$ the closed ball $\{z \in E:\|z\| \leq R\}$.

LEMma 4.3. Let $T$ be in $\Omega$. If $R$ is large enough, then

$$
A(T)=\bigcap_{n \geq 1}\left(\Phi_{T_{1}} \circ \cdots \circ \Phi_{T_{n}}\right)\left(\mathbb{B}_{R}\right) .
$$


Proof. If $n \in \mathbb{N}$ and $Q \in T_{n}$, then

$$
\|Q(z)\| \leq\|\widehat{Q}\|\|z\|+\|Q(0)\| \leq\|\widehat{T}\|\|z\|+\|T\|
$$

for any $z \in E$. Therefore if $R \geq\|T\| /(1-\|\widehat{T}\|)$, we have $Q\left(\mathbb{B}_{R}\right) \subset \mathbb{B}_{R}$ and the conclusion follows from the previous lemma.

Observe that it is enough to take

$$
R=\frac{\sup \left\{\|Q(0)\|: Q \in T_{n}, n \in \mathbb{N}\right\}}{1-\|\widehat{T}\|} \leq \frac{\|T\|}{1-\|\widehat{T}\|}
$$

in Lemma 4.3.

The last lemma allows us to give an easy example showing that not all attractors are compact.

ExAmple 4.4. Take $E=\ell^{\infty}\left(=\ell^{\infty}(\mathbb{N}, \mathbb{C})\right)$ and let $\left(e^{k}\right)_{k \in \mathbb{N}}$ be its canonical basis. Define the matrix $T=\left[T_{n, k}\right]_{n, k \in \mathbb{N}}$ by putting $T_{n, k}(z)=\frac{1}{3} z+\frac{2}{3} e^{k}$. Then $T \in \Omega_{\mathbb{N}^{2}}$, since $\|T\|=1$ and $\|\widehat{T}\|=\frac{1}{3}$. Note that $e^{k}$ is the unique fixed point of $T_{n, k}$ for each $n$ and $k$. Therefore by Lemma 4.3,

$$
\left\{e^{k}: k \in \mathbb{N}\right\} \subset \bigcap_{n \geq 1}\left(\Phi_{T_{1}} \circ \cdots \circ \Phi_{T_{n}}\right)\left(\mathbb{B}_{R}\right)=A(T)
$$

if $R \geq 1$ is large enough. Hence $A(T)$ is not compact.

Now we have two simple observations, analogous to some results from [7].

Observation 4.5. Let $T$ be in $\Omega$. Assume that all families $T_{n}$ are finite and there exists $\delta \in(0,1)$ such that

$$
\sum_{Q \in T_{n}}\|\widehat{Q}\| \leq \delta, \quad n \geq 0 .
$$

Then the attractor $A(T)$ is a singleton or is totally disconnected.

Proof. By Lemma 4.3 we can pick $R>0$ such that

$$
A(T)=\bigcap_{n \geq 1}\left(\Phi_{T_{1}} \circ \cdots \circ \Phi_{T_{n}}\right)\left(\mathbb{B}_{R}\right) .
$$

Let $S$ be a connected component of $A(T)$. For each $n$ consider the finite family

$$
\mathcal{G}_{n}:=\left\{K=\left(Q_{1} \circ \cdots \circ Q_{n}\right)\left(\mathbb{B}_{R}\right): Q_{k} \in T_{k}, k \in\{1, \ldots, n\}, K \cap S \neq \emptyset\right\} .
$$

Since $S \subset \cup \mathcal{G}_{n}$, we have

$$
\begin{aligned}
\operatorname{diam}(S) & \leq \sum_{K \in \mathcal{G}_{n}} \operatorname{diam}(K) \leq \sum_{\left(Q_{1}, \ldots, Q_{n}\right) \in T_{1} \times \cdots \times T_{n}} 2 R\left\|\widehat{Q}_{1}\right\| \cdot \ldots \cdot\left\|\widehat{Q}_{n}\right\| \\
& \leq 2 R \delta^{n} \rightarrow 0,
\end{aligned}
$$

hence $S$ is a singleton. 
For $z \in \mathbb{C}$ consider

$h_{1}(z)=\frac{3}{8} z, h_{2}(z)=\frac{3}{8} z+\frac{5}{8}, h_{3}(z)=\frac{3}{8} z+\frac{5}{8} i, h_{4}(z)=\frac{9}{64} z+\frac{9}{64}+\frac{15}{64} i$.

It is shown in [3] that the attractor of a matrix $T \in \ell^{\infty}(\mathbb{N} \times\{1,2,3,4\}, \mathcal{A}(\mathbb{C}))$ with $T_{n}=\left\{h_{1}, h_{2}, h_{3}, h_{4}\right\}, n \in \mathbb{N}$, is totally disconnected. Therefore even for matrices with finite rows the assumption about $\delta$ in Observation 4.5 is only sufficient, but not necessary.

Recall the definition of the $k$-dimensional Hausdorff measure $\mathcal{H}^{k}$ and Hausdorff dimension $\operatorname{dim}_{\mathcal{H}}$. For every $k \geq 0, \delta>0$ and $K \subset E$ we define

$$
\begin{aligned}
\mathcal{H}_{\delta}^{k}(K) & =\inf \left\{\sum_{i=0}^{\infty}\left(\operatorname{diam}\left(K_{i}\right)\right)^{k}: K \subset \bigcup_{i=0}^{\infty} K_{i}, \operatorname{diam}\left(K_{i}\right) \leq \delta\right\}, \\
\mathcal{H}^{k}(K) & =\lim _{\delta \rightarrow 0} \mathcal{H}_{\delta}^{k}(K)=\sup _{\delta \geq 0} \mathcal{H}_{\delta}^{k}(K), \\
\operatorname{dim}_{\mathcal{H}}(K) & =\sup \left\{k \geq 0: \mathcal{H}^{k}(K)=\infty\right\}=\inf \left\{k \geq 0: \mathcal{H}^{k}(K)=0\right\} .
\end{aligned}
$$

The following observation will be proved only for $T \in \Omega$ such that all families $T_{n}$ are finite. For a fixed $n$ we define

$$
\phi_{n}:[0, \infty) \ni t \mapsto \phi_{n}(t)=\sum_{Q \in T_{n}}\|\widehat{Q}\|^{t} \in\left(0, \sharp T_{n}\right] .
$$

The function $\phi_{n}$ is strictly decreasing, continuous and $\phi_{n}(0)=\sharp T_{n} \geq 1$, hence there exists a unique $\alpha_{n} \in[0, \infty)$ with $\phi_{n}\left(\alpha_{n}\right)=1$.

ObSERVATION 4.6. If $T \in \Omega$ is such that all families $T_{n}$ are finite and $\alpha=\sup \left\{\alpha_{n}: n \in \mathbb{N}\right\}<\infty$, then $\operatorname{dim}_{\mathcal{H}}(A(T)) \leq \alpha$.

Proof. By Lemma 4.3 there exists a number $R>0$ such that for each $n$,

$$
A(T) \subset \bigcup_{\left(Q_{1}, \ldots, Q_{n}\right) \in T_{1} \times \cdots \times T_{n}}\left(Q_{1} \circ \cdots \circ Q_{n}\right)\left(\mathbb{B}_{R}\right) .
$$

We know that

$$
\operatorname{diam}\left(\left(Q_{1} \circ \cdots \circ Q_{n}\right)\left(\mathbb{B}_{R}\right)\right)=2 R\left\|\widehat{Q}_{1}\right\| \cdot \ldots \cdot\left\|\widehat{Q}_{n}\right\| \leq 2 R\|\widehat{T}\|^{n} \rightarrow 0,
$$

which means that for $n$ large enough we can use the family

$$
\mathcal{G}_{n}:=\left\{K=\left(Q_{1} \circ \cdots \circ Q_{n}\right)\left(\mathbb{B}_{R}\right): Q_{k} \in T_{k}, k \in\{1, \ldots, n\}\right\}
$$

to calculate the sum in the definition of the Hausdorff measure. Now

$$
\begin{aligned}
\sum_{K \in \mathcal{G}_{n}}(\operatorname{diam}(K))^{\alpha} & \leq \sum_{\left(Q_{1}, \ldots, Q_{n}\right) \in T_{1} \times \cdots \times T_{n}}(2 R)^{\alpha}\left\|\widehat{Q}_{1}\right\|^{\alpha} \cdot \ldots \cdot\left\|\widehat{Q}_{n}\right\|^{\alpha} \\
& =(2 R)^{\alpha} \phi_{1}(\alpha) \phi_{2}(\alpha) \cdot \ldots \cdot \phi_{n}(\alpha) \leq(2 R)^{\alpha} .
\end{aligned}
$$

Hence $\mathcal{H}^{\alpha}(A(T))<\infty$. 
We conclude this section by remarking that a sufficient condition for $\alpha$ to be finite is that $k=\sup \left\{\sharp T_{n}: n \in \mathbb{N}\right\}<\infty$, since $\phi_{n}(t) \leq k\|\widehat{T}\|^{t}$ for all $n$ and $t$ (and hence $\alpha \leq-(\log k) /(\log \|\widehat{T}\|))$.

5. Relation between attractors of matrices and sequences. In this section we restrict the investigation to two cases: either the dimension of the space $E$ is finite, or we have matrices with finite rows (in the first case we can consider $\Omega_{\mathbb{N}^{2}}$, in the second we take $\Omega_{\mathbb{N}_{\rho}}$ ). Since in both situations the attractors of matrices $T$ are compact, we can also restrict the mappings $\Phi_{T_{n}}$ to $\operatorname{Comp}(E)$ using formula (6). In what follows, $f$ and $f_{n}$ are defined as in Theorem 2.3 .

Proposition 5.1. Assume that either the dimension of the space $E$ is finite or the matrices considered have finite rows. Let $T \in \Omega$. Define

$$
\operatorname{Col}(T):=\left\{Q=\left(Q_{n}\right)_{n \geq 1}: Q_{n} \in \bar{T}_{n}, n \in \mathbb{N}\right\}
$$

and $a(T):=\{f(Q): Q \in \operatorname{Col}(T)\}$. Then $A(T)=\overline{a(T)}$.

Proof. Since for any $Q \in \operatorname{Col}(T)$ we have $\|Q\| \leq\|T\|$ and $\|\widehat{Q}\| \leq\|\widehat{T}\|$, the family $\operatorname{Col}(T)$ is a subset of $\Omega_{\mathbb{N}}$. If $K$ is in $\operatorname{Comp}(E), R=\sup \{\|z\|: z \in K\}$ and $z \in K$, we use (3) to show that

$$
\operatorname{dist}\left(f(Q), \Phi_{T_{1}}(K)\right) \leq\left\|f(Q)-f_{1}(Q, z)\right\| \leq(2 R+\|T\|) \frac{\|\widehat{T}\|}{1-\|\widehat{T}\|},
$$

for any $Q \in \operatorname{Col}(T)$. In particular, we see that the set $a(T)$ is bounded.

Fix $n \in \mathbb{N}$. Pick $\zeta \in \overline{a(T)}$ and $\varepsilon>0$. There exists $Q \in \operatorname{Col}(T)$ satisfying $\|\zeta-f(Q)\|<\varepsilon$. We can pick $w \in\left(\Phi_{T_{1}} \circ \cdots \circ \Phi_{T_{n}}\right)(K)$ with $\|f(Q)-w\|=$ $\operatorname{dist}\left(f(Q),\left(\Phi_{T_{1}} \circ \cdots \circ \Phi_{T_{n}}\right)(K)\right)$. Then by $(3)$,

$$
\begin{aligned}
\operatorname{dist}\left(\zeta,\left(\Phi_{T_{1}} \circ \cdots \circ \Phi_{T_{n}}\right)(K)\right) & \leq\|\zeta-f(Q)\|+\|f(Q)-w\| \\
& \leq \varepsilon+\operatorname{dist}\left(f(Q),\left(\Phi_{T_{1}} \circ \cdots \circ \Phi_{T_{n}}\right)(K)\right) \\
& \leq \varepsilon+\left\|f(Q)-f_{n}(Q, z)\right\| \\
& \leq \varepsilon+(2 R+\|T\|) \frac{\|\widehat{T}\|^{n}}{1-\|\widehat{T}\|} .
\end{aligned}
$$

Letting $\varepsilon$ tend to 0 we obtain

$$
\operatorname{dist}\left(\zeta,\left(\Phi_{T_{1}} \circ \cdots \circ \Phi_{T_{n}}\right)(K)\right) \leq(2 R+\|T\|) \frac{\|\widehat{T}\|^{n}}{1-\|\widehat{T}\|} .
$$

On the other hand, if $\xi \in\left(\Phi_{T_{1}} \circ \cdots \circ \Phi_{T_{n}}\right)(K)$, then there exist $\tilde{Q} \in \operatorname{Col}(T)$ and $x \in K$ such that $\xi=f_{n}(\tilde{Q}, x)$, and thus

$$
\operatorname{dist}(\xi, \overline{a(T)}) \leq\left\|f_{n}(\tilde{Q}, x)-f(\tilde{Q})\right\| \leq(2 R+\|T\|) \frac{\|\widehat{T}\|^{n}}{1-\|\widehat{T}\|}
$$

again by (3). Therefore $d_{\mathrm{H}}\left(\overline{a(T)},\left(\Phi_{T_{1}} \circ \cdots \circ \Phi_{T_{n}}\right)(K)\right) \rightarrow 0$. 
If we now take a sequence $T=\left(T_{n}\right)_{n \geq 1} \in \Omega_{\mathbb{N}}$, we can view it as a matrix with infinite constant rows. It follows from Proposition 5.1 that the attractor of the matrix is a singleton consisting of the unique limit point for the sequence, which exists by Lemma 2.2 .

Furthermore, the attractor of a matrix can have exactly $k$ points for any positive integer $k$. Moreover, if $\operatorname{dim}(E)$ is finite, then any compact subset of $E$ is the attractor of some matrix $T \in \Omega_{\mathbb{N}^{2}}$. To see this, note first that if $Q=\left(Q_{n}\right) \in \Omega_{\mathbb{N}}, k \in \mathbb{N}$ and $a \in E$ are such that $Q_{n}(a)=a$ for all $n>k$, then

$f(Q)=\lim _{n \rightarrow \infty}\left(Q_{1} \circ \cdots \circ Q_{n}\right)(a)=\lim _{n \rightarrow \infty}\left(Q_{1} \circ \cdots \circ Q_{k}\right)(a)=\left(Q_{1} \circ \cdots \circ Q_{k}\right)(a)$.

Consequently, by Proposition 5.1, if $T \in \Omega, k \in \mathbb{N}$ and $a \in E$ are such that $T_{n, j}(a)=a$ for all $n>k$ and $j$, then

$$
a(T)=\left\{\left(Q_{1} \circ \cdots \circ Q_{k}\right)(a): Q_{1} \in \bar{T}_{1}, \ldots, Q_{k} \in \bar{T}_{k}\right\} .
$$

In particular, we can obtain any finite set as an attractor. We also have the following

Example 5.2. Let diam $(E)$ be finite, $K$ be in $\operatorname{Comp}(E)$ and $\left\{a_{j}: j \in \mathbb{N}\right\}$ be an at most countable dense subset of $K$. Define

$$
\begin{array}{ll}
T_{1, j}: E \ni z \mapsto \frac{1}{2} z+a_{j} \in E, & j \in \mathbb{N}, \\
T_{n, j}: E \ni z \mapsto\left(\frac{1}{2}+\frac{1}{n+j}\right) z \in E, & j \in \mathbb{N}, n=2,3, \ldots
\end{array}
$$

Then $T=\left[T_{n, j}\right]_{n, j \in \mathbb{N}} \in \Omega_{\mathbb{N}^{2}}$ and $A(T)=\overline{a(T)}=K$.

Note that for a matrix of affine contractions of $\mathbb{C}$, if the associated attractor is finite but is not a singleton, it cannot be at the same time the attractor of an iterated function system of affine nonconstant contractions of the complex plane. Attractors of such systems are either singletons or infinite sets. Indeed, if all the functions in the system have the same fixed point then the attractor is the singleton consisting of that point. However, if two functions $h_{1}$ and $h_{2}$ in the system have distinct fixed points, say $z_{1}$ and $z_{2}$, then the invariance property of the attractor implies that all points $h_{2}^{n}\left(z_{1}\right), n \in \mathbb{N}$, are in the attractor and all these points are distinct.

6. Analyticity of attractors. Let us recall the definition of (strongly) analytic multifunction due to Słodkowski [18]. (The term "strongly analytic" is used here to make a distinction between the definition stated below and a less restrictive definition given in [17].)

Just as before, if $X$ is a metric space, $\operatorname{Comp}(X)$ denotes the set of all nonempty compact subsets of $X$. 
Let $U$ be an open set in a complex Banach space $G$. Any mapping $K$ : $U \rightarrow \operatorname{Comp}(E)$ is called a multifunction with values in $E$. By the graph of $K$ we mean the set $\operatorname{graph}(K)=\{(z, w) \in U \times E: w \in K(z)\}$. We say that a multifunction is upper semicontinuous if for any open set $W \subset E$, the set $\{z \in U: K(z) \subset W\}$ is open.

Furthermore, we say that a subset $Y$ of a complex Banach space $F$ has the local maximum property if there is no holomorphic function $f: W \rightarrow \mathbb{C}$ (where $W \subset F$ is open) such that $|f|$ restricted to $W \cap Y$ has a strict local maximum. A multifunction $K: U \rightarrow \operatorname{Comp}\left(\mathbb{C}^{N}\right)$ is said to be strongly analytic (or simply analytic) if it is upper semicontinuous and if for any $(N+1)$-dimensional complex affine subspace $L$ of $G \times \mathbb{C}^{N}$ the set $L \cap \operatorname{graph}(K)$ has the local maximum property.

Finally, we say that a multifunction is trivial analytic if its graph is the union of a family of graphs of holomorphic functions.

In this section we restrict our investigation to the space $\ell^{\infty}\left(\mathbb{N}_{\rho}, \mathcal{A}(E)\right)$ and its open subset $\Omega_{\rho}=\Omega_{\mathbb{N}_{\rho}}$. Just as in Proposition 5.1, our construction of attractors necessitates different choices of infinite sequences of entries from a matrix with an infinite number of finite rows (possibly of varying length), one entry from each row. The set $\operatorname{Col}(T)$ can be seen as a collection of somewhat crooked columns and it turns out that it can be furnished with a useful topological structure.

Let $\Sigma_{\rho}$ denote the set of all functions $\sigma: \mathbb{N} \rightarrow \mathbb{N}$ such that $\sigma \leq \rho$. Choose a nondecreasing function $\bar{\rho}: \mathbb{N} \rightarrow \mathbb{N}$ such that $\rho \leq \bar{\rho}$. We can define a metric on $\Sigma_{\rho}$ by the formula

$$
\delta(\sigma, \tau)=\sum_{j=1}^{\infty} \frac{|\sigma(j)-\tau(j)|}{\bar{\rho}(j)^{j}}, \quad \sigma, \tau \in \Sigma_{\rho} .
$$

It has been shown in [12] that for any $\sigma \in \Sigma_{\rho}$ the sets

$$
U(\sigma, m)=\left\{\tau \in \Sigma_{\rho}: \sigma(j)=\tau(j) \text { for } j=1, \ldots, m\right\}, \quad m \in \mathbb{N},
$$

form a basis of neighbourhoods of $\sigma$ and that the metric space $\left(\Sigma_{\rho}, \delta\right)$ is compact.

Suppose that $T=\left[T_{n, j}\right] \in \ell^{\infty}\left(\mathbb{N}_{\rho}, \mathcal{A}(E)\right)$. If $n \in \mathbb{N}$, then the $n$th row of $T$ will be denoted by

$$
T_{\text {row }(n)}=\left[T_{n, 1}, \ldots, T_{n, \rho(n)}\right] \in(\mathcal{A}(E))^{\rho(n)} .
$$

If $\sigma \in \Sigma_{\rho}$, then

$$
T_{\operatorname{col}(\sigma)}=\left[T_{1, \sigma(1)}, T_{2, \sigma(2)}, \ldots\right]^{*} \in \operatorname{Col}(T) \subset \ell^{\infty}(\mathbb{N}, \mathcal{A}(E)),
$$

where the asterisk denotes the transpose. If $\sigma \equiv m$, then $T_{\operatorname{col}(\sigma)}$ is simply the $m$ th column of the infinite matrix $T$. 
TheOREM 6.1. If $f$ is defined as in Theorem 2.3, then the mapping

$$
\Psi: \Sigma_{\rho} \times \Omega_{\rho} \ni(\sigma, T) \mapsto f\left(T_{\operatorname{col}(\sigma)}\right) \in E
$$

is continuous. Moreover, the set-valued mapping

$$
A: \Omega_{\rho} \ni T \mapsto A(T) \in \operatorname{Comp}(E),
$$

where $A(T)$ denotes the attractor of $T$, is upper semicontinuous. In particular, if $E=\mathbb{C}^{N}$, the mapping $A$ is a trivial strongly analytic multifunction.

Proof. The first statement follows from (3) and (8).

As for the second statement, note that if $T \in \Omega_{\rho}$, then

$$
A(T)=a(T)=\bigcup_{\sigma \in \Sigma_{\rho}}\left\{f\left(T_{\operatorname{col}(\sigma)}\right)\right\}=\Psi\left(\Sigma_{\rho} \times\{T\}\right)
$$

is a compact set in $E$.

To show the upper semicontinuity, take an open set $U \subset E$ containing $A(T)$. By the continuity of $\Psi$, for each $\sigma \in \Sigma_{\rho}$ there exists a neighbourhood $V_{\sigma} \subset \Sigma_{\rho}$ of $\sigma$ and a neighbourhood $W_{\sigma} \subset \Omega_{\rho}$ of $T$ such that $f\left(S_{\operatorname{col}(\tau)}\right) \in U$ for all $(\tau, S) \in V_{\sigma} \times W_{\sigma}$. The open cover $\left(V_{\sigma}\right)_{\sigma}$ of the compact space $\Sigma_{\rho}$ contains a finite open subcover $V_{\sigma_{1}}, \ldots, V_{\sigma_{n}}$. Let $W=W_{\sigma_{1}} \cap \cdots \cap W_{\sigma_{n}}$. If $S \in W$ and $\tau \in \Sigma_{\rho}$, then $\tau \in V_{\sigma_{i}}$ for some $\sigma_{i}$ and thus $(\tau, S) \in V_{\sigma_{i}} \times W \subset V_{\sigma_{i}} \times W_{\sigma_{i}}$. Therefore $A(S) \subset U$ as required.

The last conclusion is obvious.

7. Generalized Cantor sets. We now recall the construction of a generalized Cantor set in $\mathbb{C}$ from [15]. Let $\left(l_{n}\right)_{n \geq 0}$ be a given sequence of positive numbers such that $l_{0}=1$ and

$$
2 l_{n}<l_{n-1}
$$

for $n \geq 1$. We start with the interval $F_{0}=[0,1]$ from which we remove the open concentric subinterval of length $1-2 l_{1}$ and thus obtain the set $F_{1}=I_{1,1} \cup I_{1,2}$, where $I_{1,1}=\left[0, l_{1}\right]$ and $I_{1,2}=\left[1-l_{1}, 1\right]$. If $F_{n}=I_{n, 1} \cup I_{n, 2} \cup$ $\cdots \cup I_{n, 2^{n}}$, we obtain $F_{n+1}$ by removing the open concentric subinterval of length $l_{n}-2 l_{n+1}$ from each of the intervals $I_{n, k}$. Then

$$
F=\bigcap_{n \geq 0} F_{n}
$$

is called a generalized Cantor set.

If $l_{n}=3^{-n}(n=0,1,2, \ldots)$, we obtain the classical ternary Cantor set, probably the most popular example of attractors of iterated function systems in $\mathbb{C}$. In a slightly more general setting, if $l_{n}=a^{n}(n=0,1,2, \ldots)$ for a fixed $a \in(0,1 / 2)$, the generalized Cantor set is the attractor of the iterated function system $\left\{h_{1}, h_{2}\right\}$ with $h_{1}(z)=a z, h_{2}(z)=a z+1-a$. Barnsley [4] showed that every nonempty compact set can be approximated 
by attractors of iterated function systems. However, the sequence $\left(l_{n}\right)_{n \geq 0}$ may be such that $F$ is not the attractor of any (finite or countable) iterated function system of contractions (namely one can choose the sequence so that $F$ will satisfy the assumptions on the set $X_{1}$ in [6]). We will show though that the generalized Cantor sets defined in this section can be easily obtained as attractors of matrices in $\Omega \subset \ell^{\infty}(\mathbb{N} \times\{1,2\}, \mathcal{A}(\mathbb{C}))$.

First we need a technical lemma.

Lemma 7.1. Let $\left(l_{n}\right)_{n \geq 0}$ be a sequence of positive numbers such that $l_{0}=1$ and $2 l_{n}<l_{n-1}$ for $n \geq 1$. Define

$$
T_{n, 0}(z)=\frac{l_{n}}{l_{n-1}} z, \quad T_{n, 1}(z)=\frac{l_{n}}{l_{n-1}} z+1-\frac{l_{n}}{l_{n-1}} .
$$

Then $T_{n, j}([0,1]) \subset[0,1]$ for any $n \geq 1$ and $j \in\{0,1\}$. Furthermore, if $n \geq 1$, $\nu \in\left\{0,1, \ldots, 2^{n}-1\right\}$ and $j_{1} j_{2} \ldots j_{n}$ is the binary expansion of the number $\nu$, then

$$
\left(T_{1, j_{1}} \circ T_{2, j_{2}} \circ \cdots \circ T_{n, j_{n}}\right)(z)=l_{n} z+a_{\nu}^{n}
$$

with $0=a_{0}^{n}<a_{0}^{n}+l_{n}<a_{1}^{n}<a_{1}^{n}+l_{n}<\cdots<a_{2^{n}-1}^{n}<a_{2^{n}-1}^{n}+l_{n}=1$. Moreover, $a_{2 k+1}^{n}-a_{2 k}^{n}-l_{n}=l_{n-1}-2 l_{n}$.

Proof. The first statement follows from (9). To prove the rest we use induction with respect to $n$.

Proposition 7.2. Let $\left(l_{n}\right)_{n \geq 0}$ be a sequence of positive numbers such that $l_{0}=1$ and $2 l_{n}<l_{n-1}$ for $n \geq 1$. Then $T=\left[T_{n, j-1}\right]_{n \in \mathbb{N}, j \in\{1,2\}} \in \Omega \subset$ $\ell^{\infty}(\mathbb{N} \times\{1,2\}, \mathcal{A}(\mathbb{C}))$ where $T_{n, j-1}$ are given by formulas (11). Furthermore, $A(T)$ is the generalized Cantor set given by (10).

Proof. We have $\|T\|=1$ and $\|\widehat{T}\| \leq 1 / 2$ by definition of $T$ and (9). The second statement follows from Lemmas 4.2 and 7.1.

To finish this section, we remark that the family of the generalized Cantor sets considered here plays an important role in the constructive theory of functions (see for instance [15] and [5]).

Acknowledgements. This article was mostly written during reciprocal visits of the authors at the Jagiellonian University, Kraków, Poland and at Uppsala University, Uppsala, Sweden. The authors wish to thank the mathematics departments at both universities for their hospitality.

We would also like to thank the anonymous referee for his/her suggestions.

The research of both authors was partially supported by the G. S. Magnuson Foundation of the Royal Swedish Academy of Science. The second author was also supported in part by a grant of the Faculty of Mathematics and Computer Science of the Jagiellonian University. 


\section{References}

[1] L. Baribeau, D. Brunet, T. Ransford and J. Rostand, Iterated function systems, capacity and Green's functions, Comput. Methods Funct. Theory 4 (2004), 47-58.

[2] L. Baribeau and T. J. Ransford, Meromorphic multifunctions in complex dynamics, Ergodic Theory Dynam. Systems 12 (1992), 39-52.

[3] L. Baribeau and M. Roy, Analytic multifunctions, holomorphic motions and Hausdorff dimension in IFSs, Monatsh. Math. 147 (2006), 199-217.

[4] M. Barnsley, Fractals Everywhere, Academic Press, Boston, MA, 1988.

[5] L. Białas-Cież, Equivalence of Markov's property and Hölder continuity of the Green function for Cantor-type sets, East J. Approx. 1 (1995), 249-253.

[6] S. Crovisier and M. Rams, IFS attractors and Cantor sets, Topology Appl. 153 (2006), 1849-1859.

[7] J. E. Hutchinson, Fractals and self-similarity, Indiana Univ. Math. J. 30 (1981), $713-747$.

[8] M. Klimek, Joint spectra and analytic set-valued functions, Trans. Amer. Math. Soc. 294 (1986), 187-196.

[9] —, Iteration of analytic multifunctions, Nagoya Math. J. 162 (2001), 19-40.

[10] -, On perturbations of pluriregular sets generated by sequences of polynomial maps, Ann. Polon. Math. 80 (2003), 171-184.

[11] M. Klimek and M. Kosek, Composite Julia sets generated by infinite polynomial arrays, Bull. Sci. Math. 127 (2003), 885-897.

[12] - , - Strong analyticity of partly filled-in composite Julia sets, Set-Valued Anal. 14 (2006), 55-68.

[13] T. Nishino, Sur les ensembles pseudoconcaves, J. Math. Kyoto Univ. 1 (1962), 225245.

[14] K. Oka, Note sur les familles des fonctions analytiques multiformes etc., J. Sci. Hiroshima Univ. 4 (1934), 93-98.

[15] W. Pleśniak, A Cantor regular set which does not have Markov's property, Ann. Polon. Math. 51 (1990), 39-46.

[16] T. J. Ransford, Open mapping, inversion and implicit function theorems for analytic multivalued functions, Proc. London Math. Soc. 49 (1984), 537-562.

[17] Z. Słodkowski, Analytic set-valued functions and spectra, Math. Ann. 256 (1981), 363-386.

[18] - Analytic multifunctions, q-plurisubharmonic functions and uniform algebras, in: Proc. Conf. Banach Algebras and Several Complex Variables, F. Greenleaf and D. Gulick (eds.), Contemp. Math. 32, Amer. Math. Soc., Providence, RI, 1984, $243-258$.

[19] - An analytic set-valued selection and its applications to the corona theorem, to polynomial hulls and joint spectra, Trans. Amer. Math. Soc. 294 (1986), 367-377.

[20] - Analytic perturbation of the Taylor spectrum, ibid. 297 (1986), 319-336.

[21] - Approximation of analytic multifunctions, Proc. Amer. Math. Soc. 105 (1989), 387-396.

[22] H. Yamaguchi, Sur une uniformité des surfaces constantes d'une fonction entière de deux variables complexes, J. Math. Kyoto Univ. 13 (1973), 417-433. 
[23] H. Yamaguchi, Sur le mouvement des constantes de Robin, ibid. 15 (1975), 53-71.

Department of Mathematics

Uppsala University

P.O. Box 480

751-06 Uppsala, Sweden

E-mail: maciej.klimek@math.uu.se
Institute of Mathematics Jagiellonian University Łojasiewicza 6 30-348 Kraków, Poland E-mail: Marta.Kosek@im.uj.edu.pl

Received 21.8.2008

and in final form 7.12.2008 
